Rev. Mat. Iberoamericana 19 (2003), 179-194

\title{
Small global solutions and the nonrelativistic limit for the nonlinear Dirac equation
}

Shuji Machihara, Kenji Nakanishi and Tohru Ozawa

\begin{abstract}
In this paper we study the Cauchy problem for the nonlinear Dirac equation in the Sobolev space $H^{s}$. We prove the existence and uniqueness of global solutions for small data in $H^{s}$ with $s>1$. The method of proof is based on the Strichartz estimate of $L_{t}^{2}$ type for Dirac and Klein-Gordon equations. We also prove that the solutions of the nonlinear Dirac equation after modulation of phase converge to the corresponding solutions of the nonlinear Schrödinger equation as the speed of light tends to infinity.
\end{abstract}

\section{Introduction}

We consider the Cauchy problem for the nonlinear Dirac equation (NLD)

$$
\begin{aligned}
i \partial_{t} \psi+i c \alpha \nabla \psi-c^{2} \beta \psi+2 \lambda(\beta \psi \mid \psi) \beta \psi & =0, \\
\psi(0) & =\psi_{0},
\end{aligned}
$$

where $\psi$ is a function from $\mathbb{R}^{4}$ to $\mathbb{C}^{4}$ of the variables $(x, t) \in \mathbb{R}^{3} \times \mathbb{R}$ with $x=\left(x_{1}, x_{2}, x_{3}\right) \in \mathbb{R}^{3}, \partial_{t}=\partial / \partial t, \nabla=\left(\partial_{1}, \partial_{2}, \partial_{3}\right), \partial_{j}=\partial / \partial x_{j}, c>0, \lambda \in \mathbb{C}$. We follow the standard notation of relativistic quantum mechanics. The $\alpha_{j}$ 's and $\beta$ are $4 \times 4$ Hermitian matrices satisfying anticommutation relations, i.e. $\alpha_{j} \alpha_{k}+\alpha_{k} \alpha_{j}=2 \delta_{j k} I$, where $\delta_{j k}$ is Kronecker's delta and $I$ is the $4 \times 4$ unit matrix, $\alpha_{j} \beta+\beta \alpha_{j}=0$, and $\beta^{2}=I . \alpha \nabla$ stands for $\sum_{j=1}^{3} \alpha_{j} \partial_{j} .(\cdot \mid \cdot)$ denotes the Hermitian product in $\mathbb{C}^{4}$.

2000 Mathematics Subject Classification: 35L70

Keywords: Nonlinear Dirac equation, Strichartz's estimate, nonrelativistic limit, nonlinear Schrödinger equation. 
Our first purpose in this paper is to show the global existence of solutions of NLD for small data. Escobedo and Vega [5] have studied the local and global existence of solutions with more general forms of nonlinearity, while they remarked that they had not gained the global result for the cubic nonlinearity as above which is supposedly the most important model in relativistic quantum fields (see [4], [6], [22], [23], [24]). In fact, in [5] the global existence for solutions has been proved for (1.1) with nonlinearity replaced by $2 \lambda|(\beta \psi \mid \psi)|^{(p-1) / 2} \beta \psi$ with $p>3$. On the cubic nonlinearity problem $p=3$, the difficulty there consisted in that the Strichartz estimates of $L_{t}^{2}$ type were unknown. Here we give an affirmative answer to this question in the subcritical framework of Sobolev spaces. We construct the $L_{t}^{2}$ type Strichartz estimate for Dirac and Klein-Gordon equations. The Strichartz estimates which play on $L_{t}^{2}$ have been studied by Lindblad and Sogge [13], Ginible and Velo [9] for wave equation and by Keel and Tao [10] for wave and Schrödinger equations. We follow the argument of Keel and Tao [10] which concludes the better estimate than we need. Although we obtain the Strichartz estimate involving the end point, it is not necessarily required for our proof since the problem we will consider is subcritical one. We employ it as only $L_{t}^{2}$ type estimate.

To state the main results precisely, we introduce the following notation. For any $r$ with $1 \leq r \leq \infty, L^{r}=L^{r}\left(\mathbb{R}^{n}\right)$ denotes the Lebesgue space on $\mathbb{R}^{n}$. For any $s \in \mathbb{R}$ and any $r$ with $1<r<\infty, H_{r}^{s}$ denotes the inhomogeneous Sobolev space. For any $s \in \mathbb{R}$ and any $r, m$ with $1 \leq r, m \leq \infty, B_{r, m}^{s}$ denotes the inhomogeneous Besov space. We refer to [1] for notation and general information on these spaces. We shall work in the three dimensional space $\mathbb{R}^{3}$ and with functions with values in $\mathbb{C}^{4}$ except Lemma 3 below. We make abbreviations such as $H^{s}=H_{2}^{s}, B_{r}^{s}=B_{r, 2}^{s}$. With the notation above, we state our first result.

Theorem 1 Let $s>1$. Let $\psi_{0} \in H^{s}$ and let $\left\|\psi_{0}\right\|_{H^{s}}$ be sufficiently small. Then there exists a unique solution $\psi$ for (1.1) such that

$$
\psi \in C\left(\mathbb{R} ; H^{s}\right) \cap L^{2}\left(\mathbb{R} ; B_{r}^{s-\sigma}\right) \cap L^{\infty}\left(\mathbb{R} ; H^{s}\right),
$$

where $1 / r=1 / 2-1 /(2+\theta), \sigma=1 / 2+1 /(2+\theta)=((4+\theta) / 2)(1 / 2-1 / r)$ with sufficiently small $\theta>0$.

Moreover, there exist unique $\psi_{ \pm} \in H^{s}$ such that

$$
\lim _{t \rightarrow \pm \infty}\left\|\psi(t)-U(t) \psi_{ \pm}\right\|_{H^{s}}=0
$$

where $U(t)$ denotes the Dirac group, which solves the free Dirac equation. 
The case $s=1$ is actually the critical case for the cubic nonlinearity from the viewpoint of the scaling argument, and nothing is known even for local solutions. The difficulty is due to the lack of Strichartz estimates on $L_{t}^{2} L^{\infty}$, which is also related to the ill-posedness results for nonlinear wave equations of Lindblad [11], [12]. The case $s>1$ falls within the subcritical case for the cubic nonlinearity. Theorem 1 ensures global existence of small solutions in the subcritical case, which has been left open since [5].

Our second purpose is to study the nonrelativistic limit of NLD as $c \rightarrow \infty$. We will show that solutions of NLD after modulation of phase converge to the corresponding solutions of a coupled system of nonlinear Schrödinger type equations as the speed of light tends to infinity. Substitution by $u=2 e^{i t \beta c^{2}} \beta \psi$ into the (1.1) yields the modified nonlinear Dirac equation (mNLD)

$$
\begin{aligned}
\partial_{t} u-c e^{2 i c^{2} t \beta} \alpha \nabla u & =-\frac{i \lambda}{2}(\beta u \mid u) \beta u, \\
u(0) & =\phi_{c},
\end{aligned}
$$

where $\phi_{c}$ is equal to $2 \beta \psi_{0}$ in this situation though from now on it is convenient to regard $\phi_{c}$ as new Cauchy data depending on $c$. As Najman [19] has observed, if the Cauchy data $\phi_{c}$ converges to $\phi_{\infty}$, say, as $c \rightarrow \infty$, then the solution $u_{c}$ of (1.4) is expected to converge to a function $v: \mathbb{R}^{4} \rightarrow \mathbb{C}^{4}$ which satisfies the following nonlinear Schrödinger type equation (NLS)

$$
\begin{aligned}
\partial_{t} v-\frac{i \beta}{2} \Delta v & =-\frac{i \lambda}{2}(\beta v \mid v) \beta v, \\
v(0) & =\phi_{\infty} .
\end{aligned}
$$

There are a few papers on this problem. In [19] Najman proved under the assumption that $\phi_{c} \in H^{2}, \phi_{\infty} \in H^{2}$ and $\phi_{c} \rightarrow \phi_{\infty}$ in $H^{s}$ with $0 \leq s \leq 1$, the solutions $u_{c}$ of mNLD converge to the solution $v$ of NLS in $H^{s}$ locally in time. In [15] Matsuyama showed under the assumption that $\phi_{c} \in H^{2}, \phi_{\infty} \in H^{2}$ and $\phi_{c} \rightarrow \phi_{\infty}$ in $H^{2}$, the solutions $u_{c}$ converge to the solution $v$ weakly in $H^{2}$ locally in time. In [16], for general space dimensions $n$, convergence in weighted Sobolev spaces is studied locally in time in the setting of classical solutions under the assumptions of stronger regularity for Cauchy data.

In this paper we shall improve those available results by reducing the order of Sobolev spaces where $\phi_{c}, \phi_{\infty}, u_{c}(t), v(t)$ belong, down close to the optimum as regards the scaling structure of solutions. More specifically we prove the nonrelativistic convergence in the Sobolev spaces $H^{s}$ with $s>1$. 
Theorem 2 Let $s>1$, and let $\phi_{c}, \phi_{\infty} \in H^{s}$. Let $u_{c}$ be the solution of (1.4) with $u_{c}(0)=\phi_{c}$ and $v$ be the solution of $(1.5)$ with $v(0)=\phi_{\infty}$. Let $T_{c}^{*}$ and $T^{*}$ be the maximal existence times of $u_{c}$ and of $v$, respectively. If

$$
\lim _{c \rightarrow \infty}\left\|\phi_{c}-\phi_{\infty}\right\|_{H^{s}}=0
$$

then $\lim \inf _{c \rightarrow \infty} T_{c}^{*} \geq T^{*}$, and for any $T$ with $0<T<T^{*}$,

$$
\lim _{c \rightarrow \infty}\left\|u_{c}-v\right\|_{L^{\infty}\left(0, T ; H^{s}\right)}=0 .
$$

In Theorem 2 we treat both local and global solutions. Local existence of solutions has been proved in [5]. Global existence of small solutions with $T_{c}^{*}=\infty$ is proved by Theorem 1 above. Our method of proof of Theorem 2 is based on the observation of convergence of solutions from (1.4) to (1.5), rather than the previous treatment of convergence from second order equations of Klein-Gordon type to (1.5) as a singular limit. To make a direct convergence go through, we exploit the Strichartz estimates that are uniform in $c$.

\section{Proof of Theorem 1}

In this section, without loss of generality we may assume that $c=1$. The Dirac equation to be considered therefore takes the form,

$$
\partial_{t} \psi+\alpha \nabla \psi+i \beta \psi=f(\psi) \equiv 2 i \lambda(\beta \psi \mid \psi) \beta \psi \text {. }
$$

The corresponding linear Dirac equation becomes

$$
\partial_{t} \psi=-\alpha \nabla \psi-i \beta \psi
$$

Differentiating (2.1) in $t$, we obtain

$$
\partial_{t}^{2} \psi=-\alpha \nabla(-\alpha \nabla \psi-i \beta \psi)-i \beta(-\alpha \nabla \psi-i \beta \psi)=\Delta \psi-\psi,
$$

which is the linear Klein-Gordon equation, where the associated Cauchy data takes the form

$$
\left(\psi(0), \partial_{t} \psi(0)\right)=\left(\psi_{0},-\alpha \nabla \psi_{0}-i \beta \psi_{0}\right) .
$$

This implies that the solution $\psi$ of $(2.1)$ with Cauchy data $\psi_{0}$ is represented as

$$
\psi(t)=U(t) \psi_{0}
$$

where $U(t)$ is the free propagator defined on $L^{2}\left(\mathbb{R}^{3} ; \mathbb{C}^{4}\right)$ defined as

$$
U(t)=I \cos t(1-\Delta)^{1 / 2}-(\alpha \nabla+i \beta)(1-\Delta)^{-1 / 2} \sin t(1-\Delta)^{1 / 2} .
$$


Accordingly the solution $\psi$ of NLD will be studied in the form of the integral equation

$$
\psi(t)=U(t) \psi_{0}+\int_{0}^{t} U\left(t-t^{\prime}\right) f\left(\psi\left(t^{\prime}\right)\right) d t^{\prime}
$$

To treat (2.2) by a contraction argument, we study the Strichartz estimates for the operator $U(t)$. For that purpose we single out $K_{ \pm}(t)=e^{ \pm i t(1-\Delta)^{1 / 2}}$ as essential parts of $U(t)$.

Lemma 3 The space dimension is denoted by $n$. We have the estimates

$$
\begin{aligned}
\left\|K_{ \pm}(t) u\right\|_{L_{t}^{q_{1}} B_{r_{1}}^{-s_{1}}} & \lesssim\|u\|_{L^{2}}, \\
\left\|\int_{t^{\prime}<t} K_{ \pm}\left(t-t^{\prime}\right) f\left(t^{\prime}\right) d t^{\prime}\right\|_{L_{t}^{q_{2} B_{r_{2}}^{-s_{2}}}} & \lesssim\|f\|_{L_{t}^{q_{3}^{\prime} B_{r_{3}^{\prime}}^{s_{3}}}} .
\end{aligned}
$$

Here $2 / q_{j}=(n-1+\theta)\left(1 / 2-1 / r_{j}\right), 2 s_{j}=(n+1+\theta)\left(1 / 2-1 / r_{j}\right)$ for $0 \leq \theta \leq 1,2 \leq q_{j}, r_{j} \leq \infty,\left(q_{j}, r_{j}\right) \neq(2, \infty), j=1,2,3$, and $p^{\prime}$ denotes the dual exponent to $p$ defined by $1 / p+1 / p^{\prime}=1$.

Remark. For the case $n \geq 3$, the pair of exponents $(1 / q, 1 / r)$ with $q=2$, or equivalently, $1 / r=1 / 2-1 /(n-1+\theta) \equiv 1 / r_{e}$ is called "end point", since this case is just excluded from the standard duality argument on the Strichartz estimates [8], [13], [25]. We want to use the Lemma 3 in the case $n=3, q=2$ so that we are faced with the restriction $0<\theta \leq 1$, which is caused by $(q, r) \neq(2, \infty)$.

Proof of Lemma 3. Regarding the free Schrödinger and wave equations, the end point estimates were proved by Keel and Tao [10]. Here we follow almost the same argument with necessary modifications. Below we concentrate our attention on the end point estimate and therefore we take $n \geq 3$. We abbreviate $K_{ \pm}(t)$ to $K(t)$ for simplicity.

For (2.3), by a duality argument it is sufficient to prove that

$$
\left\|\int_{-\infty}^{\infty} K\left(-t^{\prime}\right) F\left(t^{\prime}\right) d t^{\prime}\right\|_{L^{2}} \lesssim\|F\|_{L_{t}^{q^{\prime}} B_{r^{\prime}}^{s}}
$$

which follows if we can show that

$$
\left\|\int_{-\infty}^{\infty} K\left(-t^{\prime}\right) \varphi_{k} * F\left(t^{\prime}\right) d t^{\prime}\right\|_{L^{2}} \lesssim 2^{k s}\left\|\varphi_{k} * F\right\|_{L_{t}^{q^{\prime}} L^{r^{\prime}}}
$$

where $\left\{\varphi_{k}\right\}_{0}^{\infty}$ is the Littlewood-Paley dyadic decomposition on $\mathbb{R}^{n}$ and $q, r, s$ are as in Lemma 3. 
184 S. Machinara, K. Nakanishi and T. Ozawa

Indeed, (2.5) follows from (2.6) as

$$
\begin{aligned}
\left\|\int_{-\infty}^{\infty} K\left(-t^{\prime}\right) F\left(t^{\prime}\right) d t^{\prime}\right\|_{L^{2}} & \lesssim\left(\sum_{k=0}^{\infty}\left\|\int_{-\infty}^{\infty} K\left(-t^{\prime}\right) \varphi_{k} * F\left(t^{\prime}\right) d t^{\prime}\right\|_{L^{2}}^{2}\right)^{1 / 2} \\
& \lesssim\left\|2^{k s} \varphi_{k} * F\right\|_{l^{2} L_{t}^{q^{\prime}} L^{r^{\prime}}} \\
& \lesssim\left\|2^{k s} \varphi_{k} * F\right\|_{L_{t}^{q^{\prime}} 2^{2} L^{r^{\prime}}} \\
& =\|F\|_{L_{t}^{q^{\prime}} B_{r^{\prime}}^{s}}
\end{aligned}
$$

where we have used Minkowski's inequality with $q^{\prime} \leq 2$.

Firstly we prove (2.6) for non-endpoint. We use the decay estimate for $K(t)[2],[7],[20]$

$$
\left\|K(t) \varphi_{k} * f\right\|_{L^{r}} \lesssim|t|^{-(n-1+\theta)(1 / 2-1 / r)} 2^{(n+1+\theta)(1 / 2-1 / r) k}\left\|\varphi_{k} * f\right\|_{L^{r^{\prime}}}
$$

with $0 \leq \theta \leq 1,2 \leq r \leq \infty$. From the Hardy-Littlewood-Sobolev inequality we have for $q>2$,

$$
\begin{array}{rl}
\| \int_{-\infty}^{\infty} K\left(t-t^{\prime}\right) \varphi_{k} * & F\left(t^{\prime}\right) d t^{\prime} \|_{L_{t}^{q} L^{r}} \\
& \lesssim 2^{2 k s}\left\|\int_{-\infty}^{\infty}\left|t-t^{\prime}\right|^{-2 \sigma(1 / 2-1 / r)}\right\| \varphi_{k} * F\left(t^{\prime}\right)\left\|_{L^{r^{\prime}}} d t^{\prime}\right\|_{L_{t}^{q}} \\
& \lesssim 2^{2 k s}\left\|\varphi_{k} * F\right\|_{L_{t}^{q^{\prime}} L^{r^{\prime}}} .
\end{array}
$$

Thus we obtain

$$
\begin{array}{rl}
\| \int_{-\infty}^{\infty} K\left(-t^{\prime}\right) \varphi_{k} * & F\left(t^{\prime}\right) d t^{\prime} \|_{L^{2}}^{2} \\
& =\int_{-\infty}^{\infty}\left\langle\int_{-\infty}^{\infty} K\left(t-t^{\prime}\right) \varphi_{k} * F\left(t^{\prime}\right) d t^{\prime}, \varphi_{k} * F(t)\right\rangle d t \\
& \lesssim\left\|\int_{-\infty}^{\infty} K\left(t-t^{\prime}\right) \varphi_{k} * F\left(t^{\prime}\right) d t^{\prime}\right\|_{L_{t}^{q} L^{r}}\left\|\varphi_{k} * F\right\|_{L_{t}^{q^{\prime}} L^{r^{\prime}}} \\
& \lesssim 2^{2 k s}\left\|\varphi_{k} * F\right\|_{L_{t}^{q^{\prime}} L^{r^{\prime}}}^{2},
\end{array}
$$

which is precisely (2.6) for $q>2$. 
For the estimate at end point, we consider following bilinear estimate which guarantees (2.6) by duality argument,

$$
\left|T\left(\varphi_{k} * F, G\right)\right| \lesssim 2^{2 k s}\|F\|_{L_{t}^{q^{\prime}} L^{r^{\prime}}}\|G\|_{L_{t}^{q^{\prime}} L^{r^{\prime}}}
$$

with

$$
T(f, g)=\int_{t^{\prime}<t}\left\langle K\left(-t^{\prime}\right) f\left(t^{\prime}\right), K(-t) g(t)\right\rangle d t^{\prime} d t .
$$

This is further reduced to

$$
\sum_{j=-\infty}^{\infty}\left|T_{j}\left(\varphi_{k} * F, G\right)\right| \lesssim 2^{2 k s}\|F\|_{L_{t}^{q^{\prime}} L^{r^{\prime}}}\|G\|_{L_{t}^{q^{\prime}} L^{r^{\prime}}}
$$

with

$$
T_{j}(f, g)=\int_{t-2^{j+1}<t^{\prime} \leq t-2^{j}}\left\langle K\left(-t^{\prime}\right) f\left(t^{\prime}\right), K(-t) g(t)\right\rangle d t^{\prime} d t .
$$

We show the following inequalities,

$$
\begin{aligned}
\left|T_{j}\left(\varphi_{k} * F, G\right)\right| \lesssim 2^{k \alpha(\infty, \infty)-j \beta(\infty, \infty)}\|F\|_{L_{t}^{2} L^{1}}\|G\|_{L_{t}^{2} L^{1}}, \\
\left|T_{j}\left(\varphi_{k} * F, G\right)\right| \lesssim 2^{k \alpha(a, 2)-j \beta(a, 2)}\|F\|_{L_{t}^{2} L^{a^{\prime}}}\|G\|_{L_{t}^{2} L^{2}}, \\
\left|T_{j}\left(\varphi_{k} * F, G\right)\right| \lesssim 2^{k \alpha(2, b)-j \beta(2, b)}\|F\|_{L_{t}^{2} L^{2}}\|G\|_{L_{t}^{2} L^{b^{\prime}}},
\end{aligned}
$$

where $2 \leq a, b<r_{e}, \alpha(a, b)=\lambda(1-1 / a-1 / b), \lambda=(n+1+\theta) / 2, \beta(a, b)=$ $\sigma-1-\sigma / a-\sigma / b, \sigma=(n-1+\theta) / 2$.

For the first inequality we estimate

$$
\begin{aligned}
T_{j}\left(\varphi_{k} * F, G\right) & \lesssim \int_{t-2^{j+1}<t^{\prime} \leq t-2^{j}}\left\|K\left(t-t^{\prime}\right) \varphi_{k} * F\left(t^{\prime}\right)\right\|_{L^{\infty}}\|G(t)\|_{L^{1}} d t^{\prime} d t \\
& \lesssim 2^{k \lambda} \int_{t-2^{j+1}<t^{\prime} \leq t-2^{j}}\left|t-t^{\prime}\right|^{-\sigma}\left\|\varphi_{k} * F\left(t^{\prime}\right)\right\|_{L^{1}}\|G(t)\|_{L^{1}} d t^{\prime} d t \\
& \lesssim 2^{k \lambda-j(\sigma-1)}\|F\|_{L_{t}^{2} L^{1}}\|G\|_{L_{t}^{2} L^{1}}
\end{aligned}
$$

We use non-endpoint estimate for the second inequality,

$$
\begin{aligned}
\left|T_{j}\left(\varphi_{k} * F, G\right)\right| & \lesssim\left(\int_{-\infty}^{\infty}\left\|\int_{t-2^{j+1}}^{t-2^{j}} K\left(-t^{\prime}\right) \varphi_{k} * F\left(t^{\prime}\right) d t^{\prime}\right\|_{L^{2}}^{2} d t\right)^{1 / 2}\|G\|_{L_{t}^{2} L^{2}} \\
& \lesssim 2^{s k}\left(\int_{-\infty}^{\infty}\left\|\varphi_{k} * F\right\|_{L^{q^{\prime}}\left(t-2^{j+1}, t-2^{j} ; L^{r^{\prime}}\right)}^{2} d t\right)^{1 / 2}\|G\|_{L_{t}^{2} L^{2}} \\
& \lesssim 2^{s k+j / q^{\prime}}\|F\|_{L_{t}^{2} L^{r^{\prime}}}\|G\|_{L_{t}^{2} L^{2}} .
\end{aligned}
$$

By symmetry the third inequality also follows. 
186 S. Machihara, K. NAKanishi and T. OzaWA

By interpolation between these estimates, we have for all $(1 / a, 1 / b)$ in a neighborhood of $\left(1 / r_{e}, 1 / r_{e}\right)$

$$
\left|T_{j}\left(\varphi_{k} * F, G\right)\right| \lesssim 2^{k \alpha(a, b)-j \beta(a, b)}\|F\|_{L_{t}^{2} L^{a^{\prime}}}\|G\|_{L_{t}^{2} L^{b^{\prime}}}
$$

where, for the existence of a neighborhood of $\left(1 / r_{e}, 1 / r_{e}\right)$ the point $(q, r)=$ $(2, \infty)$ is excluded.

We regard (2.11) as

$$
\begin{aligned}
\left|T_{j}\left(2^{-k \lambda} \varphi_{k} * F, G\right)\right| & \lesssim 2^{-j \beta(a, b)}\left\|2^{-k \lambda / a} \varphi_{k} * F\right\|_{L_{t}^{2} L^{a^{\prime}}}\left\|2^{-k \lambda / b} G\right\|_{L_{t}^{2} L^{b^{\prime}}} \\
& =2^{-j \beta(a, b)}\|F\|_{L_{t}^{2} 2^{k \lambda / a} L^{a^{\prime}}}\|G\|_{L_{t}^{2} 2^{k \lambda / b} L^{b^{\prime}}}
\end{aligned}
$$

We use the bilinear interpolation which is found as in Lemma 6.1 in [10]. We use the real interpolation spaces $\left(A_{0}, A_{1}\right)_{\vartheta, q}$ for $0<\vartheta<1,1 \leq q \leq \infty$, for reference [1].

We use the space

$$
i_{q}^{s}=\left\{\left\{a_{j}\right\}_{-\infty}^{\infty} \in l^{q}(\mathbb{Z}) ;\left\|\left\{a_{j}\right\}_{-\infty}^{\infty}\right\|_{i_{q}^{s}}=\left\|\left\{2^{s j} a_{j}\right\}_{-\infty}^{\infty}\right\|_{l^{q}(\mathbb{Z})}<\infty\right\} .
$$

We define

$$
T^{k}=\left\{T_{j}\left(2^{-k \lambda} \varphi_{k} * \cdot, \cdot\right)\right\}_{j=-\infty}^{\infty} .
$$

We take $a_{0} \neq a_{1}, b_{0} \neq b_{1}$ such that

$$
\beta\left(a_{0}, b_{1}\right)=\beta\left(a_{1}, b_{0}\right) \equiv \beta_{1}, \beta\left(a_{0}, b_{0}\right) \equiv \beta_{0}, \beta_{0} \neq \beta_{1},
$$

to obtain the following boundedness of $T^{k}$ as

$T^{k}:\left(L_{t}^{2} 2^{k \lambda / a_{0}} L^{a_{0}^{\prime}}, L_{t}^{2} 2^{k \lambda / a_{1}} L^{a_{1}^{\prime}}\right)_{\vartheta_{0}, 2} \times\left(L_{t}^{2} 2^{k \lambda / b_{0}} L^{b_{0}^{\prime}}, L_{t}^{2} 2^{k \lambda / b_{1}} L^{b_{1}^{\prime}}\right)_{\vartheta_{1}, 2} \rightarrow\left(i_{\infty}^{\beta_{0}}, i_{\infty}^{\beta_{1}}\right)_{\vartheta, 1}$.

We use the interpolation space identities

$$
\left(2^{s_{1}} L^{p_{1}}, 2^{s_{2}} L^{p_{2}}\right)_{\vartheta, q}=2^{s_{3}}\left(L^{p_{1}}, L^{p_{2}}\right)_{\vartheta, q}=2^{s_{3}} L^{p_{3}, q}
$$

where $s_{3}=(1-\vartheta) s_{1}+\vartheta s_{2}, 1 / p_{3}=(1-\vartheta) / p_{1}+\vartheta / p_{2}$, and $L^{p, q}$ denotes the Lorentz space. Thus we obtain the boundedness of $T^{k}$ as

$$
T^{k}: L_{t}^{2} 2^{k \lambda / a_{\vartheta_{0}}} L^{a_{\vartheta_{0}}^{\prime}, 2} \times L_{t}^{2} 2^{k \lambda / b_{\vartheta_{1}}} L^{b_{\vartheta_{1}}^{\prime}, 2} \rightarrow i_{1}^{\beta_{\vartheta}}
$$

where $1 / a_{\vartheta_{0}}=\left(1-\vartheta_{0}\right) / a_{0}+\vartheta_{0} / a_{1}, 1 / b_{\vartheta_{1}}=\left(1-\vartheta_{1}\right) / b_{0}+\vartheta_{1} / b_{1}, \beta_{\vartheta}=$ $(1-\vartheta) \beta_{\vartheta_{0}}+\vartheta \beta_{\vartheta_{1}}, \vartheta=\vartheta_{0}+\vartheta_{1}$, which imply $\beta_{\vartheta}=\beta\left(a_{\vartheta_{0}}, b_{\vartheta_{1}}\right)$. 
Applying this to $a_{\vartheta_{0}}=b_{\vartheta_{1}}=r_{e}$ and using the fact that $L^{r^{\prime}} \subset L^{r^{\prime}, 2}$ for $r^{\prime} \leq 2$, we obtain the boundedness

$$
T^{k}: L_{t}^{2} 2^{k \lambda / r_{e}} L^{r_{e}^{\prime}} \times L_{t}^{2} 2^{k \lambda / r_{e}} L^{r_{e}^{\prime}} \rightarrow l_{1}^{0}
$$

which is precisely (2.10) in the end point case. We note that $\alpha\left(r_{e}, r_{e}\right)=$ $(n+1+\theta)\left(1 / 2-1 / r_{e}\right)$.

For the retarded estimate (2.4), we start from (2.9). By duality argument,

$$
\left\|\int_{t^{\prime}<t} K\left(t^{\prime}-t\right) 2^{-k s} \varphi_{k} * F\left(t^{\prime}\right) d t^{\prime}\right\|_{L_{t}^{q} L^{r}} \lesssim\left\|2^{k s} \varphi_{k} * F\right\|_{L_{t}^{q^{\prime}} L^{r^{\prime}}}
$$

From Minkowski's inequality for $q \geq 2$ and $q^{\prime} \leq 2$, we obtain

$$
\left\|\int_{t^{\prime}<t} K\left(t^{\prime}-t\right) 2^{-k s} \varphi_{k} * F\left(t^{\prime}\right) d t^{\prime}\right\|_{L_{t}^{q} l^{2} L^{r}} \lesssim\left\|2^{k s} \varphi_{k} * F\right\|_{L_{t}^{q^{\prime}} l^{2} L^{r^{\prime}}}
$$

which implies

$$
\left\|\int_{t^{\prime}<t} K\left(t^{\prime}-t\right) F\left(t^{\prime}\right) d t^{\prime}\right\|_{L_{t}^{q} B_{r}^{-s}} \lesssim\|F\|_{L_{t}^{q^{\prime} B_{r^{\prime}}^{s}}} .
$$

Thus we have (2.4) for the case $q_{2}=q_{3}$. We estimate $T(F, G)$ to gain another cases. From (2.5) we have

$$
\begin{aligned}
|T(F, G)| & =\left|\int_{-\infty}^{\infty}\left\langle\int_{-\infty}^{t} K\left(-t^{\prime}\right) F\left(t^{\prime}\right) d t^{\prime}, K(-t) G(t)\right\rangle d t\right| \\
& \leq\left(\sup _{t}\left\|\int_{-\infty}^{t} K\left(-t^{\prime}\right) F\left(t^{\prime}\right) d t^{\prime}\right\|_{L^{2}}\right)\|G\|_{L_{t}^{1} L^{2}} \\
& =\left(\sup _{t}\left\|\int_{-\infty}^{\infty} K\left(-t^{\prime}\right) \mathcal{X}_{(-\infty, t]}\left(t^{\prime}\right) F\left(t^{\prime}\right) d t^{\prime}\right\|_{L^{2}}\right)\|G\|_{L_{t}^{1} L^{2}} \\
& \lesssim\left(\sup _{t}\left\|\mathcal{X}_{(-\infty, t]} F\right\|_{L_{t}^{q^{\prime}} B_{r^{\prime}}^{s}}\right)\|G\|_{L_{t}^{1} L^{2}} \\
& \lesssim\|F\|_{L_{t}^{q^{\prime}} B_{r^{\prime}}^{s}}\|G\|_{L_{t}^{1} L^{2}},
\end{aligned}
$$

where $\mathcal{X}_{I}$ denotes the characteristic function on the interval $I$. By symmetry we have

$$
|T(F, G)| \lesssim\|F\|_{L_{t}^{1} L^{2}}\|G\|_{L_{t}^{q^{\prime} B_{r^{\prime}}^{s}}}
$$


188 S. Machihara, K. NAKANishi And T. OzaWA

We have from duality argument,

$$
\begin{aligned}
\left\|\int_{t^{\prime}<t} K\left(t^{\prime}-t\right) F\left(t^{\prime}\right) d t^{\prime}\right\|_{L_{t}^{\infty} L^{2}} & \lesssim\|F\|_{L_{t}^{q^{\prime}} B_{r^{\prime}}^{s}}, \\
\left\|\int_{t^{\prime}<t} K\left(t^{\prime}-t\right) F\left(t^{\prime}\right) d t^{\prime}\right\|_{L_{t}^{q} B_{r}^{-s}} & \lesssim\|F\|_{L_{t}^{1} L^{2}} .
\end{aligned}
$$

By interpolating between two inequalities from (2.19) (2.20) and (2.21), we have (2.4) for all cases.

Now we prove Theorem 1 . We apply the contraction mapping principle to obtain global solutions with small data. For $s>1$ and $M>0$, we define the complete metric space $X_{M}^{s}$ as

$$
\begin{gathered}
X_{M}^{s}=\left\{\psi \in L^{\infty}\left(\mathbb{R} ; H^{s}\right) \cap L^{2}\left(\mathbb{R} ; B_{r}^{s-\sigma}\right) ;\|\psi\|_{X^{s}} \leq M\right\} \\
\|\psi\|_{X^{s}}=\|\psi\|_{L^{\infty}\left(\mathbb{R} ; H^{s}\right)}+\|\psi\|_{L^{2}\left(\mathbb{R} ; B_{r}^{s-\sigma}\right)},
\end{gathered}
$$

where $1 / r=1 / 2-1 /(2+\theta), \sigma=((4+\theta) / 2)(1 / 2-1 / r)$ with $0<\theta \leq 1$ to be determined later.

We show that the map $A$, given by

$$
(A(\psi))(t)=U(t) \psi_{0}+\int_{0}^{t} U\left(t-t^{\prime}\right) f\left(\psi\left(t^{\prime}\right)\right) d t^{\prime},
$$

is a contraction on $X_{M}^{s}$. From the estimates (2.3), (2.4), we have

$$
\begin{aligned}
\|A \psi\|_{X^{s}} \lesssim & \left\|\psi_{0}\right\|_{H^{s}}+\left\|(\alpha \nabla+i \beta)(1-\Delta)^{-1 / 2} \psi_{0}\right\|_{H^{s}} \\
& +\|f(\psi)\|_{L_{t}^{1} H^{s}}+\left\|(\alpha \nabla+i \beta)(1-\Delta)^{-1 / 2} f(\psi)\right\|_{L_{t}^{1} H^{s}} \\
\lesssim & \left\|\psi_{0}\right\|_{H^{s}}+\|f(\psi)\|_{L_{t}^{1} H^{s}}
\end{aligned}
$$

For $f(\psi)=2 i \lambda(\beta \psi \mid \psi) \beta \psi$, we estimate

$$
\begin{aligned}
\|f(\psi)\|_{L_{t}^{1} H^{s}} & \lesssim\|\psi\|_{L_{t}^{2} L^{\infty}}^{2}\|\psi\|_{L_{t}^{\infty} H^{s}} \\
& \lesssim\|\psi\|_{L_{t}^{2} B_{r}^{s-\sigma}}^{2}\|\psi\|_{L_{t}^{\infty} H^{s}} \lesssim\|\psi\|_{X^{s}}^{3}
\end{aligned}
$$

where the second inequality follows from the embedding

$$
B_{r}^{s-\sigma} \subset L^{\infty}
$$

provided $0>3 / r-(s-\sigma)=\theta /(\theta+2)+1-s$ with $\theta>0$ sufficiently small. This yields

$$
\begin{aligned}
\|A \psi\|_{X^{s}} & \leq C_{1}\left\|\psi_{0}\right\|_{H^{s}}+C_{2}\|\psi\|_{X^{s}}^{3} \\
& \leq C_{1}\left\|\psi_{0}\right\|_{H^{s}}+C_{2} M^{3} .
\end{aligned}
$$


Similarly, we have

$$
\begin{aligned}
\left\|f\left(\psi_{1}\right)-f\left(\psi_{2}\right)\right\|_{L_{t}^{1} H^{s}} & \lesssim\left(\left\|\psi_{1}\right\|_{X^{s}}^{2}+\left\|\psi_{2}\right\|_{X^{s}}^{2}\right)\left\|\psi_{1}-\psi_{2}\right\|_{X^{s}} \\
& \lesssim M^{2}\left\|\psi_{1}-\psi_{2}\right\|_{X^{s}}
\end{aligned}
$$

This yields

$$
\left\|A \psi_{1}-A \psi_{2}\right\|_{X^{s}} \leq C_{3} M^{2}\left\|\psi_{1}-\psi_{2}\right\|_{X^{s}} .
$$

By (2.27) and (2.28), if the $H^{s}$ norm of the data is so small that $M$ is chosen to satisfy

$$
C_{1}\left\|\psi_{0}\right\|_{H^{s}}+C_{2} M^{3} \leq M, \quad C_{3} M^{2}<1
$$

then $A$ is a contraction map on $X_{M}^{s}$ to have a unique fixed point there.

The rest of the theorem follows by the standard argument.

\section{Proof of Theorem 2}

We study (1.4) in the integral form as

$$
u_{c}(t)=V_{c}(t) \phi_{c}+\int_{0}^{t} V_{c}(t) V_{c}\left(t^{\prime}\right)^{-1} f\left(u_{c}\left(t^{\prime}\right)\right) d t^{\prime},
$$

where

$V_{c}(t)=e^{i t c^{2} \beta}\left\{I \cos t\left(c^{4}-c^{2} \Delta\right)^{1 / 2}+\left(c \alpha \nabla-i c^{2} \beta\right)\left(c^{4}-c^{2} \Delta\right)^{-1 / 2} \sin t\left(c^{4}-c^{2} \Delta\right)^{1 / 2}\right\}$.

To prove Theorem 2 on the basis of (3.1), we need the Strichartz estimates for $V_{c}(t)$ with explicit dependence on the parameter $c$. Since $V_{c}(t)$ is a linear combination of $V_{ \pm}(t)=e^{ \pm i t\left(c^{4}-c^{2} \Delta\right)^{1 / 2}}$ with bounded Fourier multipliers, it suffices to have such estimates for $V_{ \pm}(t)$, which can be found in $[14$, Lemma 2.1]. We rewrite a subset of the estimates for reader's convenience.

Lemma 4 Let $n=3$. Then

$$
\begin{gathered}
\left\|V_{ \pm}(t)|\nabla|^{-1 / q_{1}}\left(1-c^{-2} \Delta\right)^{-1 /\left(2 q_{1}\right)} u\right\|_{L_{t}^{q_{1} L^{r_{1}}}} \lesssim\|u\|_{L^{2}} \\
\left\|\int_{t^{\prime}<t} V_{ \pm}\left(t-t^{\prime}\right)|\nabla|^{-1 / q_{2}}\left(1-c^{-2} \Delta\right)^{-1 /\left(2 q_{2}\right)} f\left(t^{\prime}\right) d t^{\prime}\right\|_{L_{t}^{q_{2}} L^{r_{2}}} \\
\lesssim\left\||\nabla|^{1 / q_{3}}\left(1-c^{-2} \Delta\right)^{1 /\left(2 q_{3}\right)} f\right\|_{L_{t}^{q_{3}^{\prime}} L^{r_{3}^{\prime}}},
\end{gathered}
$$

where $1 / q_{j}=1 / 2-1 / r_{j}, 2<q_{j} \leq \infty, j=1,2,3$, and $|\nabla|^{\gamma}=(-\Delta)^{\gamma / 2}$. 
Local existence of solutions for NLD has been proved in [5]. We shall prove the uniform boundedness of solutions with respect to $c$. For $2<q \leq \infty$, we define

$$
Y_{q}^{s}=L^{q}\left(0, T ; H_{r}^{s-2 / q}\right)
$$

where $1 / r=1 / 2-1 / q$. For any $2<q \leq \infty$, we apply (3.2) to have

$$
\left\|V_{c}(t) \phi\right\|_{Y_{q}^{s}} \lesssim\|\phi\|_{H^{s}}+\left\||\nabla|^{1 / q}\left(1-c^{-2} \Delta\right)^{1 /(2 q)}(1-\Delta)^{-1 / q} u\right\|_{H^{s}} \lesssim\|\phi\|_{H^{s}}
$$

From an elementary calculation we have

$$
\begin{aligned}
V_{c}(t) V_{c}\left(t^{\prime}\right)^{-1}= & e^{i\left(t-t^{\prime}\right) c^{2} \beta}\left\{I \cos \left(t-t^{\prime}\right)\left(c^{4}-c^{2} \Delta\right)^{1 / 2}\right. \\
& \left.-i c^{2} \beta\left(c^{4}-c^{2} \Delta\right)^{-1 / 2} \sin \left(t-t^{\prime}\right)\left(c^{4}-c^{2} \Delta\right)^{1 / 2}\right\} \\
& +e^{i\left(t+t^{\prime}\right) c^{2} \beta} c \alpha \nabla\left(c^{4}-c^{2} \Delta\right)^{-1 / 2} \sin \left(t-t^{\prime}\right)\left(c^{4}-c^{2} \Delta\right)^{1 / 2}
\end{aligned}
$$

Thus we use (3.3) to obtain

$$
\left\|\int_{0}^{t} V_{c}(t) V_{c}\left(t^{\prime}\right)^{-1} f\left(t^{\prime}\right) d t^{\prime}\right\|_{Y_{q}^{s}} \lesssim\|f\|_{L_{t}^{1} H^{s}}
$$

Therefore we have from (3.1)

$$
\begin{aligned}
\left\|u_{c}\right\|_{Y_{q}^{s}} & \lesssim\left\|\phi_{c}\right\|_{H^{s}}+\left\|f\left(u_{c}\right)\right\|_{L_{t}^{1} H^{s}} \\
& \lesssim\left\|\phi_{c}\right\|_{H^{s}}+T^{1-2 / \tilde{q}}\left\|u_{c}\right\|_{L_{t}^{\tilde{q}} L_{x}^{\infty}}^{2}\left\|u_{c}\right\|_{L_{t}^{\infty} H^{s}},
\end{aligned}
$$

for any $2<q \leq \infty$.

Since we have $Y_{\tilde{q}}^{s} \subset L_{t}^{\tilde{q}} L^{\infty}$ when $\tilde{q}>2$ is sufficiently close to 2 , the above estimate and (1.6) imply

$$
\sup _{c>1}\left\|u_{c}\right\|_{Y_{q}^{s}} \lesssim \sup _{c>1}\left\|\phi_{c}\right\|_{H^{s}}<\infty
$$

for sufficiently small $T$ and for any $2<q \leq \infty$.

We follow the same procedure for the integral equation associated with (1.5) replacing $v$ by $u_{\infty}$

$$
u_{\infty}(t)=V_{\infty}(t) \phi_{\infty}+\frac{i \beta}{2} \int_{0}^{t} V_{\infty}\left(t-t^{\prime}\right) f\left(u_{\infty}\left(t^{\prime}\right)\right) d t^{\prime},
$$

where

$$
V_{\infty}(t)=e^{\frac{i}{2} t \beta \Delta}
$$

obtaining the same estimate $u_{\infty} \in Y_{q}^{s}$ and also $f\left(u_{\infty}\right) \in L_{t}^{1} H^{s}$. 
We are now in a position to prove Theorem 2. We subtract (3.9) from (3.1), and divide the result into four parts as

$$
u_{c}(t)-u_{\infty}(t)=\sum_{j=1}^{4} P_{c}^{(j)}(t)
$$

where

$$
\begin{aligned}
P_{c}^{(1)}(t) & =\left(V_{c}(t)-V_{\infty}(t)\right) \phi_{\infty}, \\
P_{c}^{(2)}(t) & =V_{c}(t)\left(\phi_{c}-\phi_{\infty}\right), \\
P_{c}^{(3)}(t) & =\int_{0}^{t}\left(V_{c}(t) V_{c}\left(t^{\prime}\right)^{-1}-V_{\infty}\left(t-t^{\prime}\right)\right) f\left(u_{\infty}\left(t^{\prime}\right)\right) d t^{\prime}, \\
P_{c}^{(4)}(t) & =\int_{0}^{t} V_{c}(t) V_{c}\left(t^{\prime}\right)^{-1}\left(f\left(u_{c}\left(t^{\prime}\right)\right)-f\left(u_{\infty}\left(t^{\prime}\right)\right)\right) d t^{\prime} .
\end{aligned}
$$

Since $\phi_{\infty} \in H^{s}$, we deduce

$$
\left\|P_{c}^{(1)}\right\|_{L_{t}^{\infty} H^{s}} \rightarrow 0
$$

from the strong convergence of $V_{c}(t)$ to $V_{\infty}(t)$ on $H^{s}$, which can be easily verified by the dominated convergence theorem in the Fourier space and the pointwise convergence of the symbol

$$
\begin{aligned}
\tilde{V}_{c}(t) & =e^{i t c^{2} \beta}\left\{\cos t\left(c^{4}+c^{2}|\xi|^{2}\right)^{1 / 2}-\frac{i c \alpha \xi+i c^{2} \beta}{\left(c^{4}+c^{2}|\xi|^{2}\right)^{1 / 2}} \sin t\left(c^{4}+c^{2}|\xi|^{2}\right)^{1 / 2}\right\} \\
& =e^{i t c^{2} \beta}\left(\cos t\left(c^{4}+c^{2}|\xi|^{2}\right)^{1 / 2}-i \beta \sin t\left(c^{4}+c^{2}|\xi|^{2}\right)^{1 / 2}\right)+o(1) \\
& =e^{i t\left(c^{2}-\left(c^{4}+c^{2}|\xi|^{2}\right)^{1 / 2}\right) \beta}+o(1)=e^{-i t|\xi|^{2} \beta / 2}+o(1) .
\end{aligned}
$$

We also have the following convergence,

$$
\begin{aligned}
\tilde{V}_{c}(t)^{-1} & =\tilde{V}_{c}(-t)+\left(e^{-i t c^{2} \beta}-e^{i t c^{2} \beta}\right) \frac{i c \alpha \xi}{\left(c^{4}+c^{2}|\xi|^{2}\right)^{1 / 2}} \sin t\left(c^{4}+c^{2}|\xi|^{2}\right)^{1 / 2} \\
& =\tilde{V}_{c}(-t)+o(1)=\tilde{V}_{\infty}(-t)+o(1) .
\end{aligned}
$$

We obtain from $f\left(u_{\infty}\right) \in L_{t}^{1} H^{s}$,

$$
\left\|P_{c}^{(3)}\right\|_{L_{t}^{\infty} H^{s}} \rightarrow 0 .
$$

From assumption (1.6), we have

$$
\left\|P_{c}^{(2)}\right\|_{L_{t}^{\infty} H^{s}} \rightarrow 0
$$


For any $2<q \leq \infty$, we estimate $P_{c}^{(4)}$ as

$$
\begin{aligned}
\left\|P_{c}^{(4)}\right\|_{Y_{q}^{s}} \lesssim & \left\|f\left(u_{c}\right)-f\left(u_{\infty}\right)\right\|_{L_{t}^{1} H^{s}} \\
\lesssim & \left(\left\|u_{c}\right\|_{L_{t}^{2} L^{\infty}}^{2}+\|v\|_{L_{t}^{2} L^{\infty}}^{2}\right)\left\|u_{c}-u_{\infty}\right\|_{L_{t}^{\infty} H^{s}} \\
& +\left(\left\|u_{c}\right\|_{L_{t}^{2} L^{\infty}}\left\|u_{c}\right\|_{L_{t}^{\infty} H^{s}}+\left\|u_{\infty}\right\|_{L_{t}^{2} L^{\infty}}\left\|u_{\infty}\right\|_{L_{t}^{\infty} H^{s}}\right)\left\|u_{c}-u_{\infty}\right\|_{L_{t}^{2} L^{\infty}} \\
& \vdots T^{1-2 / \tilde{q}}\left(\left\|u_{c}\right\|_{Y_{\tilde{q}}^{s} \cap Y_{\infty}^{s}}^{2}+\left\|u_{\infty}\right\|_{Y_{\tilde{q}}^{s} \cap Y_{\infty}^{s}}^{2}\right)\left\|u_{c}-u_{\infty}\right\|_{Y_{\tilde{q}}^{s} \cap Y_{\infty}^{s}},
\end{aligned}
$$

where $\tilde{q}>2$ is sufficiently close to 2 .

We define $\mathcal{P}_{c}=\sum_{j=1}^{3} P_{c}^{(j)}$ namely $u_{c}-u_{\infty}=\mathcal{P}_{c}+P_{c}^{(4)}$. This estimate together with the uniform bound on $u_{c}$ and $u_{\infty}$ implies that $\mathcal{P}_{c}$ is also bounded in $Y_{q}^{s}$. Moreover we already know

$$
\left\|\mathcal{P}_{c}\right\|_{L_{t}^{\infty} H^{s}} \rightarrow 0
$$

We have interpolation estimates

$$
\left\|\mathcal{P}_{c}\right\|_{Y_{q_{1}}^{s}} \lesssim\left\|\mathcal{P}_{c}\right\|_{L_{t}^{\infty} H^{s}}^{1-\theta}\left\|\mathcal{P}_{c}\right\|_{Y_{q_{2}}^{s}}^{\theta}
$$

with $1 / q_{1}=\theta / q_{2}, 2<q_{2}<q_{1} \leq \infty$. Therefore we have

$$
\left\|\mathcal{P}_{c}\right\|_{Y_{q}^{s}} \rightarrow 0
$$

for any $2<q \leq \infty$. Putting this and (3.20) together, we conclude that

$$
\left\|u_{c}-u_{\infty}\right\|_{Y_{q}^{s}} \rightarrow 0
$$

for any $2<q \leq \infty$ and sufficiently small $T$.

By a standard continuation argument, we finally obtain the convergence up to the maximal existence time of $u_{\infty}$.

\section{References}

[1] Bergh, J. And LÖfström, J.: Interpolation spaces. Springer, BerlinHeiderberg-New York, 1976.

[2] Brenner, P.: On scattering and everywhere defined scattering operators for nonlinear Klein-Gordon equations. J. Differential Equations 56 (1985), 310-344.

[3] Cazenave, T. and Weissler, F. B.: The Cauchy problem for the critical nonlinear Schrödinger equations in $H^{s}$. Nonlinear Anal. 14 (1990), 807-836.

[4] Dias, J. P. And Figueira, M.: Global existence of solutions with small initial data in $H^{s}$ for the massive nonlinear Dirac equations in three space dimensions. Boll. Un. Mat. Ital. B(7) 1 (1987), 861-874. 
[5] Escobedo, M. And Vega, L.: A semilinear Dirac equation in $H^{s}\left(\mathbb{R}^{3}\right)$ for $s>1$. SIAM J. Math. Anal. 2 (1997), 338-362.

[6] Finkelstein, R., Lelevier, R. And Ruderman, M.: Nonlinear spinor fields. Phys. Rev. 83 (1951), 326-332.

[7] Ginibre, J. And Velo, G.: Time decay of finite energy solutions of the non linear Klein-Gordon and Schrödinger equations. Ann. Inst. H. Poincaré Phys. Théor. 43 (1985), 399-442.

[8] Ginibre, J. AND Velo, G.: Smoothing properties and retarded estimates for some dispersive evolution equations. Commun. Math. Phys. 144 (1992), $163-188$.

[9] Ginibre, J. And Velo, G.: Generalized Strichartz inequalities for the wave equation. J. Funct. Anal. 133 (1995), 50-68.

[10] Keel, M. And TaO, T.: Endpoint Strichartz estimates. Amer. J. Math. 120 (1998), 955-980.

[11] Lindblad, H.: A sharp counterexample to local existence of low regularity solutions to nonlinear wave equations. Duke Math. J. 72 (1993), 503-539.

[12] LindBlad, H.: Counterexamples to local existence for semi-linear wave equations. Amer. J. Math. 118 (1996), 1-16.

[13] Lindblad, H. And Sogge, C. D.: On existence and scattering with minimal regularity for semilinear wave equations. J. Funct. Anal. 130 (1995), $357-426$.

[14] Machihara, S., Nakanishi, K. and Ozawa, T.: Nonrelativistic limit in the energy space for nonlinear Klein-Gordon equations. Math. Ann. 322 (2002), 603-621.

[15] Matsuyama, T.: A remark on the nonrelativistic limit for semilinear Dirac equations. Nonlinear Anal. 25 (1995), 1139-1146.

[16] Matsuyama, T.: Rapidly decreasing solutions and nonrelativistic limit of semilinear Dirac equations. Rev. Math. Phys. 7 (1995), 243-267.

[17] Najman, B.: The nonrelativistic limit of the nonlinear Klein-Gordon equation. Nonlinear Anal. 15 (1990), 217-228.

[18] Najman, B.: The nonrelativistic limit of the Klein-Gordon and Dirac equations. In Differential Equations with Applications in Biology, Physics and Engineering, J. Goldstein, F. Kappel, W. Schappacher (Eds.), Lect. Notes Pure Appl. Math. 133 (1991), 291-299, Marcel Dekker.

[19] Najman, B.: The nonrelativistic limit of the nonlinear Dirac equation. Ann. Inst. H. Poincaré, Anal. Non linéaire 9 (1992), 3-12.

[20] Pecher, H.: Nonlinear small data scattering for the wave and KleinGordon equation. Math. Z. 185 (1984), 261-270.

[21] Ponce, G. And Sideris, T. C.: Local regularity of nonlinear wave equations in three space dimensions. Comm. Partial Differential Equations 18 (1993), 169-177. 
194 S. Machihara, K. NAKAnishi And T. OzaWA

[22] RañAda, A. F.: Classical nonlinear Dirac field models of extended particles. In Quantum Theory, Groups, Fields and Particles. D. Reidel, Amsterdam, 1982.

[23] Soler, M.: Classical, stable nonlinear spinor field with positive rest energy. Phys. Rev. D 1 (1970), 2766-2769.

[24] Wakano, M.: Intensely localized solutions of the classical Dirac-Maxwell field equations. Progr. Theoret. Phys. 35 (1966), 1117-1141.

[25] WANG, B.: On existence and scattering for critical and subcritical nonlinear Klein-Gordon equation in $H^{s}$. Nonlinear Anal. 31 (1998), 573-587.

Recibido: 7 de septiembre de 2001

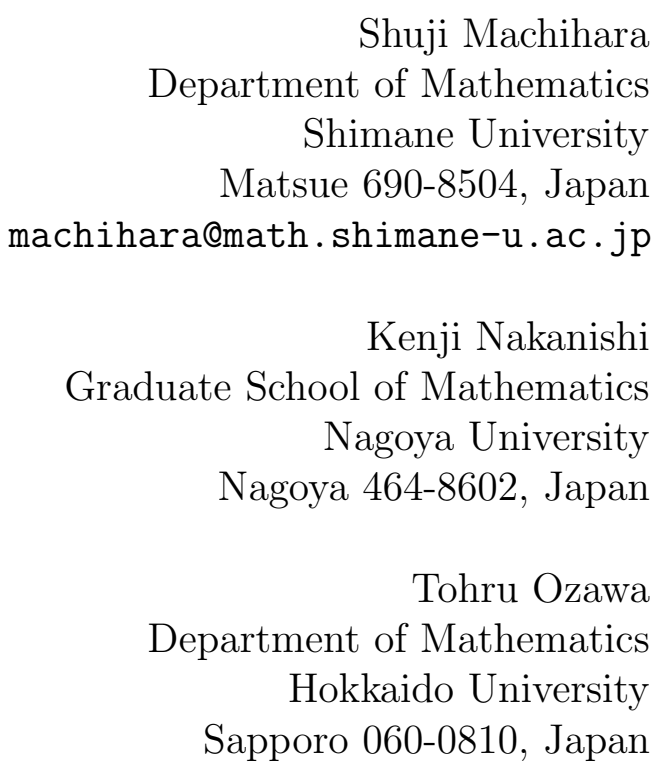

